\title{
Conservation Laws and Traveling Wave Solutions of a Generalized Nonlinear ZK-BBM Equation
}

\author{
Khadijo Rashid Adem and Chaudry Masood Khalique \\ International Institute for Symmetry Analysis and Mathematical Modelling, Department of Mathematical Sciences, \\ North-West University, Mafikeng Campus, Private Bag X 2046, Mmabatho 2735, South Africa
}

Correspondence should be addressed to Chaudry Masood Khalique; masood.khalique@nwu.ac.za

Received 25 February 2014; Accepted 3 April 2014; Published 23 April 2014

Academic Editor: Mariano Torrisi

Copyright (C) 2014 K. R. Adem and C. M. Khalique. This is an open access article distributed under the Creative Commons Attribution License, which permits unrestricted use, distribution, and reproduction in any medium, provided the original work is properly cited.

We study a generalized two-dimensional nonlinear Zakharov-Kuznetsov-Benjamin-Bona-Mahony (ZK-BBM) equation, which is in fact Benjamin-Bona-Mahony equation formulated in the ZK sense. Conservation laws for this equation are constructed by using the new conservation theorem due to Ibragimov and the multiplier method. Furthermore, traveling wave solutions are obtained by employing the $\left(G^{\prime} / G\right)$-expansion method.

\section{Introduction}

Many phenomena in the real world are often described by nonlinear evolution equations (NLEEs) and therefore such equations play an important role in applied mathematics, physics, and engineering. Unfortunately, there are no general methods for obtaining exact solutions for the NLEEs. However, various powerful methods have been developed by many authors to construct exact solutions of NLEEs. These methods include the inverse scattering transform method [1], Darboux transformation [2], Hirota's bilinear method [3], Bäcklund transformation [4], multiple expfunction method [5], the $\left(G^{\prime} / G\right)$-expansion method [6], the sine-cosine method [7], the F-expansion method [8], the expfunction expansion method [9], and Lie symmetry method [10].

In addition to exact solutions there is a need to find conservation laws for the NLEEs. Conservation laws assist in the numerical integration of partial differential equations [11], theory of nonclassical transformations [12,13], normal forms, and asymptotic integrability [14]. Recently, conservation laws have been used to derive exact solutions of partial differential equations [15-17].
In this paper, we analyze one such NLEE, namely, the generalized $(2+1)$-dimensional nonlinear Zakharov-KuznetsovBenjamin-Bona-Mahony (ZK-BBM) equation [18] that is given by

$$
u_{t}+u_{x}+a\left(u^{n}\right)_{x}+b\left(u_{x t}+u_{y y}\right)_{x}=0
$$

Here, in (1) $a, b$, and $n>1$ are real-valued constants. Several authors (see, e.g., the papers [18-23]) have studied this equation. The sine-cosine method, the tanh method, and the extended tanh method were used in $[18,19]$ and solitary solutions were obtained. Some exact solutions were obtained by Abdou [20, 21] by using the extended F-expansion method and the extended mapping method with symbolic computation. Mahmoudi et al. [22] used the exp-function method to obtain some solitary solutions and periodic solutions. Bifurcation method was used by Song and Yang [23] to obtain exact solitary wave solutions and kink wave solutions.

In this paper, conservation laws will be derived for (1) using the new conservation theorem due to Ibragimov [24] and the multiplier method [25]. Moreover, the $\left(G^{\prime} / G\right)$ expansion method [6] is used to obtain the traveling wave solutions for (1). 


\section{Conservation Laws}

In this section, we construct conservation laws for (1). The new conservation theorem due to Ibragimov [24] will be used and later we also employ the multiplier method [25]. For the notations used in this section, the reader is referred to [26].

To use the conservation theorem due to Ibragimov [24] we need to know the Lie point symmetries of (1). Thus, we first compute the symmetries of (1).

2.1. Lie Point Symmetries. The symmetry group of ZK-BBM equation (1) will be generated by

$$
\begin{aligned}
X= & \xi^{1}(x, y, t, u) \frac{\partial}{\partial x}+\xi^{2}(x, y, t, u) \frac{\partial}{\partial y} \\
& +\xi^{3}(x, y, t, u) \frac{\partial}{\partial t}+\eta(x, y, t, u) \frac{\partial}{\partial u} .
\end{aligned}
$$

Applying the third prolongation $\mathrm{pr}^{(3)} X$ to (1), we obtain the following overdetermined system of linear partial differential equations:

$$
\begin{gathered}
\xi_{y}^{3}=0, \quad \xi_{y}^{1}=0, \\
\xi_{x}^{1}=0, \quad \xi_{t}^{2}=0, \\
\xi_{x}^{2}=0, \quad \xi_{u}^{2}=0, \\
\xi_{t}^{1}=0, \quad \xi_{u}^{1}=0, \\
\xi_{x}^{3}=0, \quad \xi_{u}^{3}=0, \\
\eta_{t u}=0, \quad \eta_{x u}=0, \\
\eta_{u u}=0, \quad \xi_{t}^{3}-2 \xi_{y}^{2}=0, \\
\xi_{y y}^{2}-2 \eta_{y u}=0, \\
a n u^{n} \eta_{x}+u \eta_{x}+u \eta_{t}+b u \eta_{x x t}+b u \eta_{x y y}=0, \\
a n^{2} u^{n} \eta-a n u^{n} \eta+u^{2} \xi_{t}^{3}+a n u^{n+1} \xi_{t}^{3}+b u^{2} \eta_{y y u}=0 .
\end{gathered}
$$

Solving the above partial differential equations, one obtains the following three Lie point symmetries:

$$
X_{1}=\frac{\partial}{\partial x}, \quad X_{2}=\frac{\partial}{\partial y}, \quad X_{3}=\frac{\partial}{\partial t} .
$$

2.2. Application of the Conservation Theorem. The generalized two-dimensional nonlinear ZK-BBM equation together with its adjoint equation are given by

$$
\begin{aligned}
& E_{\alpha} \equiv u_{t}+u_{x}+a n u^{n-1} u_{x}+b\left(u_{x t}+u_{y y}\right)_{x}=0, \\
& E_{\alpha}^{*} \equiv v_{t}+v_{x}+a n u^{n-1} v_{x}+b\left(v_{x t}+v_{y y}\right)_{x}=0 .
\end{aligned}
$$

The third-order Lagrangian for the system of (5a) and (5b) is given by

$$
L=v\left(u_{t}+u_{x}+a n u^{n-1} u_{x}+b\left(u_{x t}+u_{y y}\right)_{x}\right),
$$

which can be reduced to the second-order Lagrangian:

$$
L=v\left(u_{t}+u_{x}+a n u^{n-1} u_{x}\right)-b v_{t} u_{x x}-b v_{x} u_{y y} .
$$

We have the following three cases.

(i) We first consider Lie point symmetry $X_{1}=\partial_{x}$ of (1). Corresponding to this symmetry the Lie characteristic functions are $W^{1}=-u_{x}$ and $W^{2}=-v_{x}$. Thus, by using Ibragimov theorem [24], the components of the conserved vector associated with the symmetry $X_{1}=\partial_{x}$ are given by

$$
\begin{aligned}
& T_{1}^{t}=b v_{x} u_{x x}-v u_{x}, \\
& T_{1}^{x}=v u_{t}-b u_{x} v_{t x}, \\
& T_{1}^{y}=b v_{x} u_{x y}-b u_{x} v_{x y} .
\end{aligned}
$$

(ii) Likewise, Lie point symmetry $X_{2}=\partial_{y}$ has Lie characteristic functions $W^{1}=-u_{y}$ and $W^{2}=$ $-v_{y}$. Invoking Ibragimov theorem, we obtain the conserved vector whose components are

$$
\begin{aligned}
& T_{2}^{t}=b v_{y} u_{x x}-v u_{y} \\
& T_{2}^{x}=-a n v u^{n-1} u_{y}-v u_{y}+b v_{t} u_{x y}-b u_{y} v_{t x}+b v_{y} u_{y y} \\
& T_{2}^{y}=a n v u^{n-1} u_{x}+v u_{x}+v u_{t}-b v_{t} u_{x x}-b u_{y} v_{x y} .
\end{aligned}
$$

(iii) Finally, Lie point symmetry $X_{3}=\partial_{t}$ gives $W^{1}=-u_{t}$ and $W^{2}=-v_{t}$ and so the associated conserved vector has components

$$
\begin{aligned}
& T_{1}^{t}=a n v u_{x} u^{n-1}+v u_{x}-b v_{x} u_{y y}, \\
& T_{1}^{x}=-a n v u^{n-1} u_{t}-v u_{t}+b v_{t} u_{t x}-b u_{t} v_{t x}+b v_{t} u_{y y}, \\
& T_{1}^{y}=b v_{x} u_{t y}-b u_{t} v_{x y} .
\end{aligned}
$$

2.3. Application of the Multiplier Method. The zeroth-order multiplier [25] for (1), namely, $\Lambda(t, x, y, u)$, is given by

$$
\Lambda=C u+F(y)
$$

where $C$ is a constant, and $F(y)$ is arbitrary function of $y$. Corresponding to the above multiplier, we have the following two conserved vectors of (1):

$$
\begin{gathered}
T_{1}^{t}=\frac{1}{6}\left\{2 b u u_{x x}+3 u^{2}-b u_{x}^{2}\right\} \\
T_{1}^{x}=\frac{1}{6(n+1)}\left\{2 b n u u_{y y}+4 b n u u_{t x}+2 b u u_{y y}+4 b u u_{t x}\right. \\
+6 a n u^{n+1}+3 n u^{2}+3 u^{2}-2 b n u_{t} u_{x} \\
\left.-b n u_{y}^{2}-2 b u_{t} u_{x}-b u_{y}^{2}\right\}
\end{gathered}
$$




$$
\begin{aligned}
& T_{1}^{y}= \frac{1}{3}\left\{2 b u u_{x y}-b u_{x} u_{y}\right\}, \\
& T_{2}^{t}= \frac{1}{3}\left\{3 u F(y)+b F(y) u_{x x}\right\}, \\
& T_{2}^{x}=\frac{1}{3}\left\{3 a u^{n} F(y)+b u F^{\prime \prime}(y)+3 u F(y)\right.\left.\quad-b F^{\prime}(y) u_{y}+2 b F(y) u_{t x}+b F(y) u_{y y}\right\}, \\
& T_{2}^{y}=\frac{1}{3}\left\{2 b F(y) u_{x y}-b F^{\prime}(y) u_{x}\right\} .
\end{aligned}
$$

Remark 1. Since $F(y)$ is an arbitrary function in the multiplier, we obtain an infinitely many conservation laws for (1).

\section{Exact Solutions Using $\left(G^{\prime} / G\right)$-Expansion Method}

In this section, we use the $\left(G^{\prime} / G\right)$-expansion method [6] to obtain exact solutions of the ZK-BBM equation (1) for $n=2$ and $n=3$.

Making use of the wave variable

$$
z=k_{1} x+k_{2} y+k_{3} t+k_{4}
$$

where $k_{i}, i=1, \ldots, 4$ are constants, the ZK-BBM equation (1) for $n=2$ and $n=3$ transforms to the third-order nonlinear ordinary differential equations (ODEs),

$$
\begin{gathered}
k_{3} \psi^{\prime}(z)+k_{1} \psi^{\prime}(z)+2 a k_{1} \psi(z) \psi^{\prime}(z) \\
+b k_{1}^{2} k_{3} \psi^{\prime \prime \prime}(z)+b k_{2}^{2} k_{1} \psi^{\prime \prime \prime}(z)=0, \\
k_{3} \psi^{\prime}(z)+k_{1} \psi^{\prime}(z)+3 a k_{1} \psi^{2}(z) \psi^{\prime}(z) \\
+b k_{1}^{2} k_{3} \psi^{\prime \prime \prime}(z)+b k_{2}^{2} k_{1} \psi^{\prime \prime \prime}(z)=0,
\end{gathered}
$$

respectively.

We look for solutions of (14) and (15) in the form:

$$
\psi(z)=\sum_{i=0}^{M} \mathscr{A}_{i}\left(\frac{G^{\prime}(z)}{G(z)}\right)^{i}
$$

where $G(z)$ satisfies the second-order ODE:

$$
G^{\prime \prime}+\lambda G^{\prime}+\mu G=0
$$

with $\lambda$ and $\mu$ as constants. Here, the constant $M$ will be determined by the homogeneous balance procedure between the highest order derivative and highest order nonlinear term appearing in (14) and (15). $\mathscr{A}_{0}, \ldots, \mathscr{A}_{M}$, are parameters to be determined:

$$
n=2 \text {. }
$$

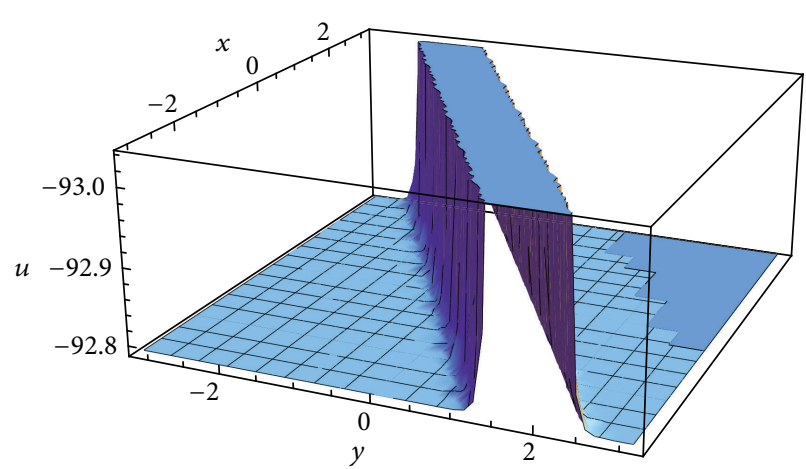

FIGURE 1: Profile of solution (21).

Application of the balancing procedure yields $M=2$ and so the solution of (14) is of the form

$$
\psi(z)=\mathscr{A}_{0}+\mathscr{A}_{1}\left(\frac{G^{\prime}(z)}{G(z)}\right)+\mathscr{A}_{2}\left(\frac{G^{\prime}(z)}{G(z)}\right)^{2} .
$$

Substituting (17) into (14) and using (19) lead to an overdetermined system of algebraic equations. Solving this system of algebraic equations, with the aid of Mathematica, we obtain

$$
\begin{aligned}
& \mathscr{A}_{0}=\frac{8 a b \mu A_{2}^{2} k_{1}^{2}+a b A_{1}^{2} k_{1}^{2}-6 b A_{2} k_{1}^{2}+6 b A_{2} k_{2}^{2}+a A_{2}^{2}}{12 a b A_{2} k_{1}^{2}}, \\
& \mathscr{A}_{1}=-\frac{\lambda\left(6 b k_{1} k_{3}+6 b k_{2}^{2}\right)}{a}, \\
& \mathscr{A}_{2}=-\frac{6 b k_{1} k_{3}+6 b k_{2}^{2}}{a} .
\end{aligned}
$$

Now, using the general solution of (17) in (19), we have the following three types of traveling wave solutions of the $\mathrm{ZK}$ BBM equation (1).

When $\lambda^{2}-4 \mu>0$, we obtain the hyperbolic function solutions:

$$
\begin{aligned}
u(x, y, t) & \\
= & \mathscr{A}_{0}+\mathscr{A}_{1}\left(-\frac{\lambda}{2}+\delta_{1} \frac{C_{1} \sinh \left(\delta_{1} z\right)+C_{2} \cosh \left(\delta_{1} z\right)}{C_{1} \cosh \left(\delta_{1} z\right)+C_{2} \sinh \left(\delta_{1} z\right)}\right) \\
& +\mathscr{A}_{2}\left(-\frac{\lambda}{2}+\delta_{1} \frac{C_{1} \sinh \left(\delta_{1} z\right)+C_{2} \cosh \left(\delta_{1} z\right)}{C_{1} \cosh \left(\delta_{1} z\right)+C_{2} \sinh \left(\delta_{1} z\right)}\right)^{2},
\end{aligned}
$$

where $z=k_{1} x+k_{2} y+k_{3} t+k_{4}, \delta_{1}=(1 / 2) \sqrt{\lambda^{2}-4 \mu}, C_{1}$, and $C_{2}$ are arbitrary constants.

The profile of solution (21) is given in Figure 1. 


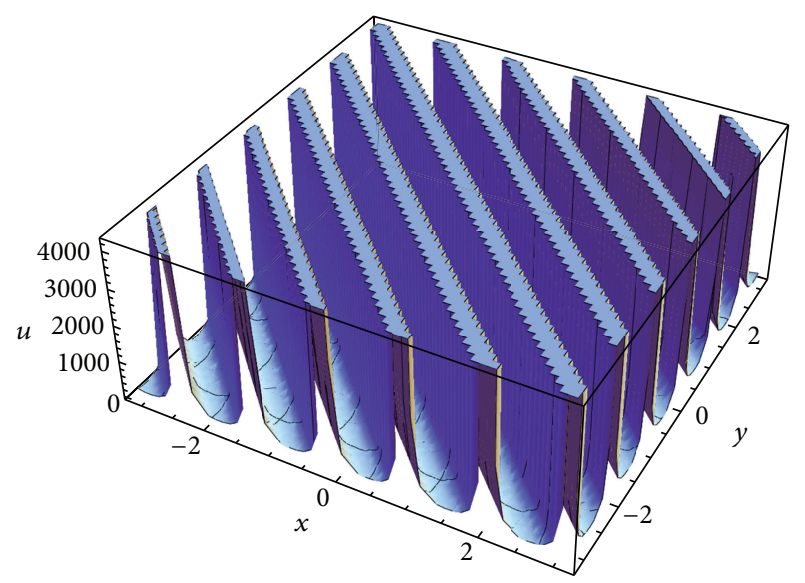

FIgURE 2: Profile of solution (22).

When $\lambda^{2}-4 \mu<0$, we obtain the trigonometric function solutions

$$
\begin{aligned}
u(x, y, t) & \\
= & \mathscr{A}_{0}+\mathscr{A}_{1}\left(-\frac{\lambda}{2}+\delta_{2} \frac{-C_{1} \sin \left(\delta_{2} z\right)+C_{2} \cos \left(\delta_{2} z\right)}{C_{1} \cos \left(\delta_{2} z\right)+C_{2} \sin \left(\delta_{2} z\right)}\right) \\
& +\mathscr{A}_{2}\left(-\frac{\lambda}{2}+\delta_{2} \frac{-C_{1} \sin \left(\delta_{2} z\right)+C_{2} \cos \left(\delta_{2} z\right)}{C_{1} \cos \left(\delta_{2} z\right)+C_{2} \sin \left(\delta_{2} z\right)}\right)^{2},
\end{aligned}
$$

where $z=k_{1} x+k_{2} y+k_{3} t+k_{4}, \delta_{2}=(1 / 2) \sqrt{4 \mu-\lambda^{2}}, C_{1}$, and $\mathrm{C}_{2}$ are arbitrary constants.

The profile of solution (22) is given in Figure 2.

When $\lambda^{2}-4 \mu=0$, we obtain the rational function solutions:

$$
\begin{aligned}
u(x, y, t)= & \mathscr{A}_{0}+\mathscr{A}_{1}\left(-\frac{\lambda}{2}+\frac{C_{2}}{C_{1}+C_{2} z}\right) \\
& +\mathscr{A}_{2}\left(-\frac{\lambda}{2}+\frac{C_{2}}{C_{1}+C_{2} z}\right)^{2},
\end{aligned}
$$

where $z=k_{1} x+k_{2} y+k_{3} t+k_{4}, C_{1}$, and $C_{2}$ are arbitrary constants.

The profile of solution (23) is given in Figure 3:

$$
n=3 .
$$

Applying the balancing procedure, in this case, we obtain $M=1$, so the solution of (15) is of the form:

$$
\psi(z)=\mathscr{A}_{0}+\mathscr{A}_{1}\left(\frac{G^{\prime}(z)}{G(z)}\right) .
$$

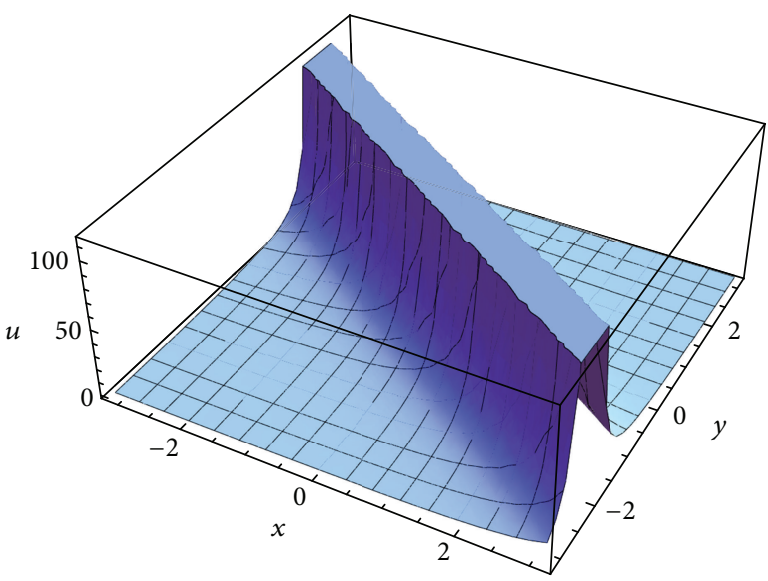

Figure 3: Profile of solution (23).

Substituting (17) into (15) and making use of (25) lead to an overdetermined system of algebraic equations, whose solution is

$$
\begin{aligned}
\mu & =\frac{-2 a A_{1}^{2}-4 b k_{2}^{2}+b k_{1}^{2} \lambda^{2} a A_{1}^{2}+4 b k_{1}^{2}}{4 a b k_{1}^{2} A_{1}^{2}}, \\
\mathscr{A}_{0} & =\frac{\lambda \sqrt{-\left(2 b k_{1} k_{3}+2 b k_{2}^{2}\right)}}{2 \sqrt{a}}, \\
\mathscr{A}_{1} & =\sqrt{\frac{-\left(2 b k_{1} k_{3}+2 b k_{2}^{2}\right)}{a}} .
\end{aligned}
$$

Consequently, as before, when $\lambda^{2}-4 \mu>0$, we obtain the hyperbolic function solutions:

$$
\begin{aligned}
u(x, y, t)= & \mathscr{A}_{0}+\mathscr{A}_{1} \\
& \times\left(-\frac{\lambda}{2}+\delta_{1} \frac{C_{1} \sinh \left(\delta_{1} z\right)+C_{2} \cosh \left(\delta_{1} z\right)}{C_{1} \cosh \left(\delta_{1} z\right)+C_{2} \sinh \left(\delta_{1} z\right)},\right.
\end{aligned}
$$

where $z=k_{1} x+k_{2} y+k_{3} t+k_{4}, \delta_{1}=(1 / 2) \sqrt{\lambda^{2}-4 \mu}, C_{1}$, and $C_{2}$ are arbitrary constants.

When $\lambda^{2}-4 \mu<0$, we obtain the trigonometric function solutions:

$$
\begin{aligned}
u(x, y, t)= & \mathscr{A}_{0}+\mathscr{A}_{1} \\
& \times\left(-\frac{\lambda}{2}+\delta_{2} \frac{-C_{1} \sin \left(\delta_{2} z\right)+C_{2} \cos \left(\delta_{2} z\right)}{C_{1} \cos \left(\delta_{2} z\right)+C_{2} \sin \left(\delta_{2} z\right)}\right),
\end{aligned}
$$

where $z=k_{1} x+k_{2} y+k_{3} t+k_{4}, \delta_{2}=(1 / 2) \sqrt{4 \mu-\lambda^{2}}, C_{1}$, and $C_{2}$ are arbitrary constants.

When $\lambda^{2}-4 \mu=0$, we obtain the rational function solutions:

$$
u(x, y, t)=\mathscr{A}_{0}+\mathscr{A}_{1}\left(-\frac{\lambda}{2}+\frac{C_{2}}{C_{1}+C_{2} z}\right),
$$

where $z=k_{1} x+k_{2} y+k_{3} t+k_{4}, C_{1}$, and $C_{2}$ are arbitrary constants. 


\section{Concluding Remarks}

In this paper, conservation laws of the generalized $(2+$ 1)-dimensional nonlinear Zakharov-Kuznetsov-BenjaminBona-Mahony equation were derived by using two different methods: the new conservation theorem and the multiplier method. Moreover, the $\left(G^{\prime} / G\right)$-expansion method was employed to obtain traveling wave solutions of the generalized $(2+1)$-dimensional Zakharov-Kuznetsov-BenjaminBona-Mahony.

\section{Conflict of Interests}

The authors declare that there is no conflict of interests regarding the publication of this paper.

\section{References}

[1] M. J. Ablowitz and P. A. Clarkson, Soliton, Nonlinear Evolution Equations and Inverse Scattering, vol. 149, Cambridge University Press, Cambridge, UK, 1991.

[2] V. B. Matveev and M. A. Salle, Darboux Transformation and Soliton, Springer, Berlin, Germany, 1991.

[3] R. Hirota, The Direct Method in Soliton Theory, vol. 155, Cambridge University Press, Cambridge, 2004.

[4] C. H. Gu, Soliton Theory and Its Application, Zhejiang Science and Technology Press, Zhejiang, China, 1990.

[5] W. X. Ma, T. Huang, and Y. Zhang, "A multiple exp-function method for nonlinear differential equations and its application," Physica Scripta, vol. 82, no. 6, Article ID 065003, 2010.

[6] M. Wang, X. Li, and J. Zhang, "The $\left(G^{\prime} / G\right)$-expansion method and travelling wave solutions of nonlinear evolution equations in mathematical physics," Physics Letters A, vol. 372, no. 4, pp. 417-423, 2008.

[7] A.-M. Wazwaz, "The tanh and the sine-cosine methods for compact and noncompact solutions of the nonlinear KleinGordon equation," Applied Mathematics and Computation, vol. 167, no. 2, pp. 1179-1195, 2005.

[8] M. Wang and X. Li, "Extended F-expansion method and periodic wave solutions for the generalized Zakharov equations," Physics Letters A, vol. 343, no. 1-3, pp. 48-54, 2005.

[9] J.-H. He and X.-H. Wu, "Exp-function method for nonlinear wave equations," Chaos, Solitons \& Fractals, vol. 30, no. 3, pp. 700-708, 2006.

[10] P. J. Olver, Applications of Lie Groups to Differential Equations, Graduate Texts in Mathematics, vol. 107, Springer, New York, NY, USA, 2nd edition, 1993.

[11] R. J. LeVeque, Numerical Methods for Conservation Laws, Birkhäuser, Basel, Switzerland, 1992.

[12] A. V. Mikhaŭlov, A. B. Shabat, and R. I. Yamilov, "On an extension of the module of invertible transformations," Doklady Akademii Nauk SSSR, vol. 295, no. 2, pp. 288-291, 1987.

[13] A. V. Mikhaǔlov, A. B. Shabat, and R. I. Yamilov, "Extension of the module of invertible transformations. Classification of integrable systems," Communications in Mathematical Physics, vol. 115, no. 1, pp. 1-19, 1988.

[14] Y. Kodama and A. V. Mikhailov, "Obstacles to asymptotic integrability," in Algebraic Aspects of Integrability, I. M. Gelfand and A. Fokas, Eds., pp. 173-204, Birkhäuser, Basel, Switzerland, 1996.
[15] A. Sjöberg, "Double reduction of PDEs from the association of symmetries with conservation laws with applications," Applied Mathematics and Computation, vol. 184, no. 2, pp. 608-616, 2007.

[16] A. Sjöberg, "On double reductions from symmetries and conservation laws," Nonlinear Analysis. Real World Applications. An International Multidisciplinary Journal, vol. 10, no. 6, pp. 34723477, 2009.

[17] A. H. Bokhari, A. Y. Al-Dweik, F. D. Zaman, A. H. Kara, and F. M. Mahomed, "Generalization of the double reduction theory," Nonlinear Analysis. Real World Applications. An International Multidisciplinary Journal, vol. 11, no. 5, pp. 3763-3769, 2010.

[18] A.-M. Wazwaz, "Compact and noncompact physical structures for the ZK-BBM equation," Applied Mathematics and Computation, vol. 169, no. 1, pp. 713-725, 2005.

[19] A.-M. Wazwaz, "The extended tanh method for new compact and noncompact solutions for the KP-BBM and the ZK-BBM equations," Chaos, Solitons and Fractals, vol. 38, no. 5, pp. 15051516, 2008.

[20] M. A. Abdou, "The extended F-expansion method and its application for a class of nonlinear evolution equations," Chaos, Solitons \& Fractals, vol. 31, no. 1, pp. 95-104, 2007.

[21] M. A. Abdou, "Exact periodic wave solutions to some nonlinear evolution equations," International Journal of Nonlinear Science, vol. 6, no. 2, pp. 145-153, 2008.

[22] J. Mahmoudi, N. Tolou, I. Khatami, A. Barari, and D. D. Ganji, "Explicit solution of nonlinear ZK-BBM wave equation using Exp-function method," Journal of Applied Sciences, vol. 8, no. 2, pp. 358-363, 2008.

[23] M. Song and C. Yang, "Exact traveling wave solutions of the Zakharov-Kuznetsov-Benjamin-Bona-Mahony equation," Applied Mathematics and Computation, vol. 216, no. 11, pp. 3234-3243, 2010.

[24] N. H. Ibragimov, "A new conservation theorem," Journal of Mathematical Analysis and Applications, vol. 333, no. 1, pp. 311328, 2007.

[25] S. C. Anco and G. Bluman, "Direct construction method for conservation laws of partial differential equations. I. Examples of conservation law classifications," European Journal of Applied Mathematics, vol. 13, no. 5, pp. 545-566, 2002.

[26] A. R. Adem and C. M. Khalique, "On the solutions and conservation laws of a coupled KdV system," Applied Mathematics and Computation, vol. 219, no. 3, pp. 959-969, 2012. 


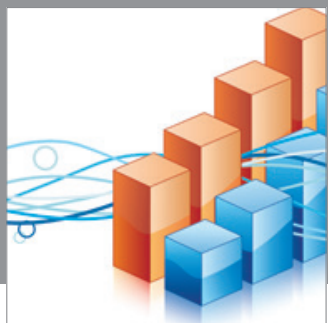

Advances in

Operations Research

mansans

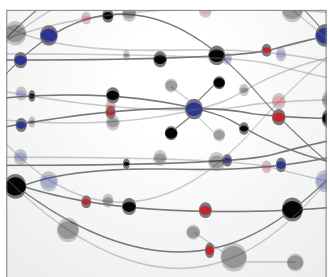

The Scientific World Journal
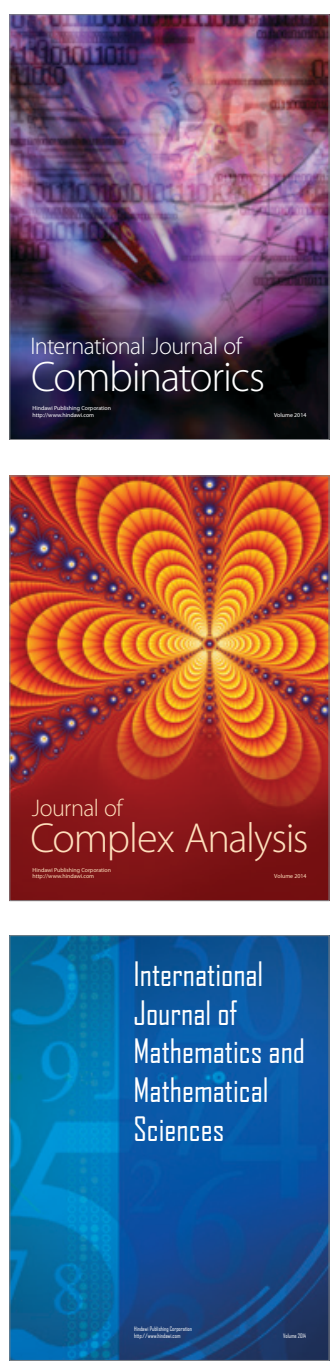
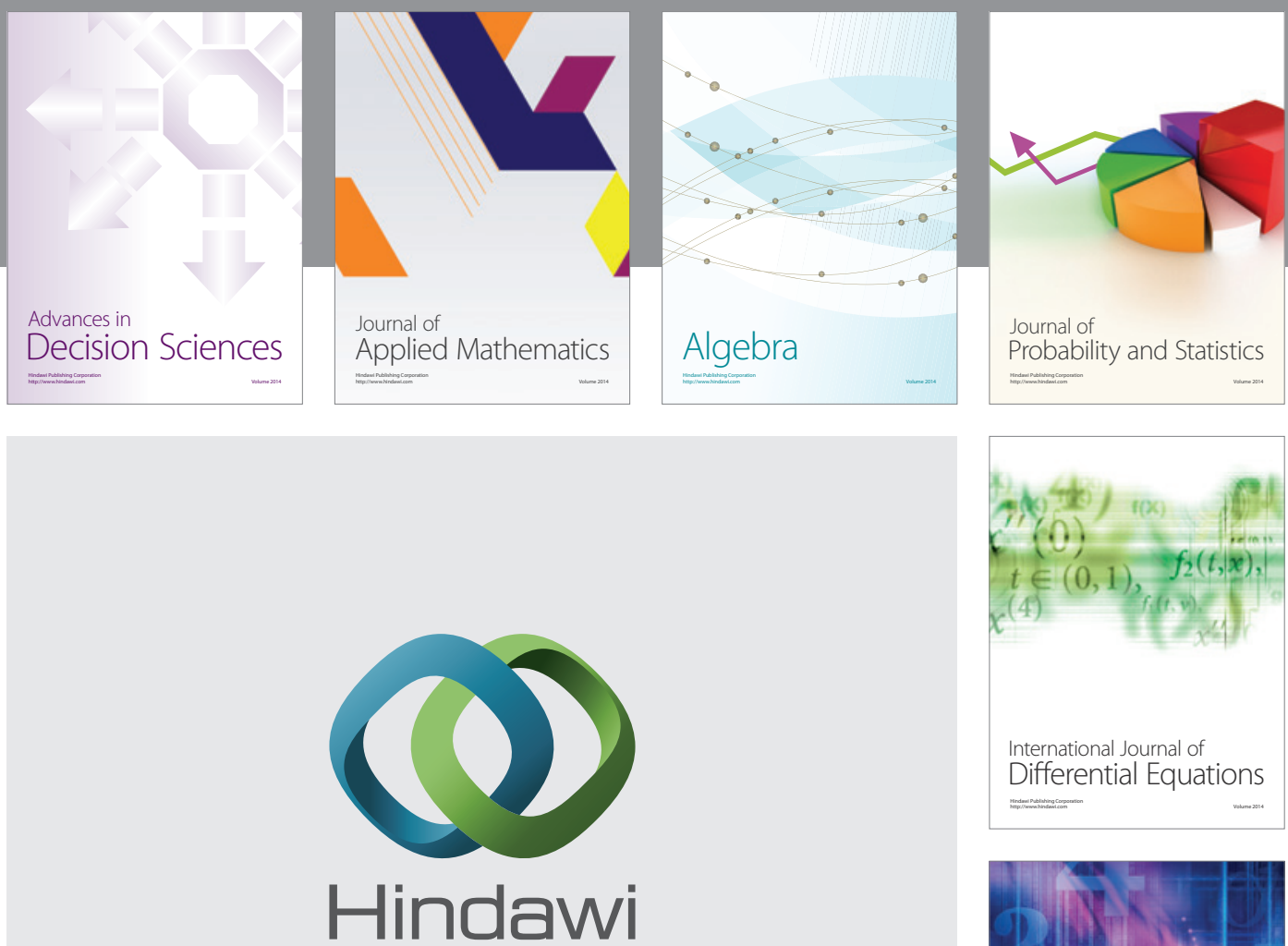

Submit your manuscripts at http://www.hindawi.com
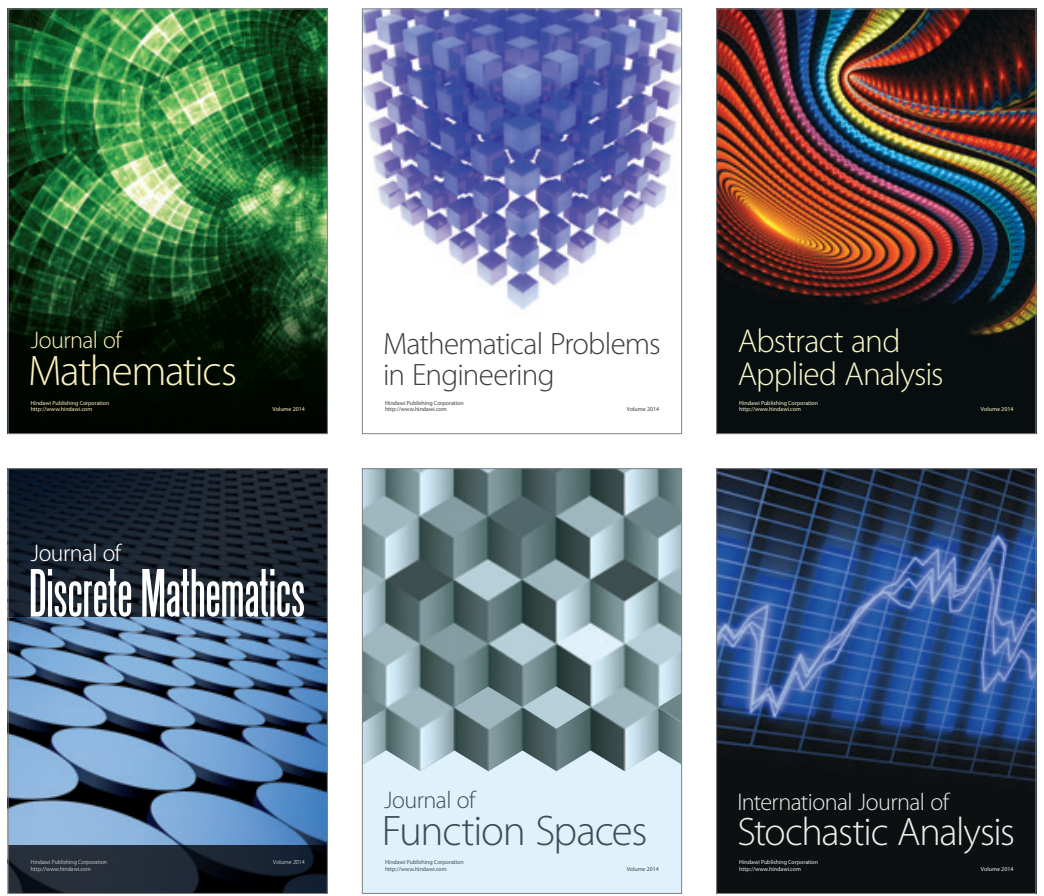

Journal of

Function Spaces

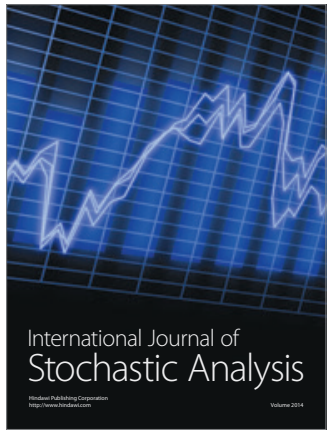

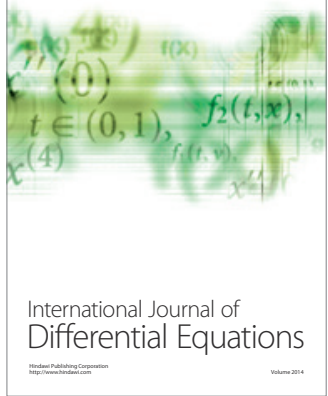
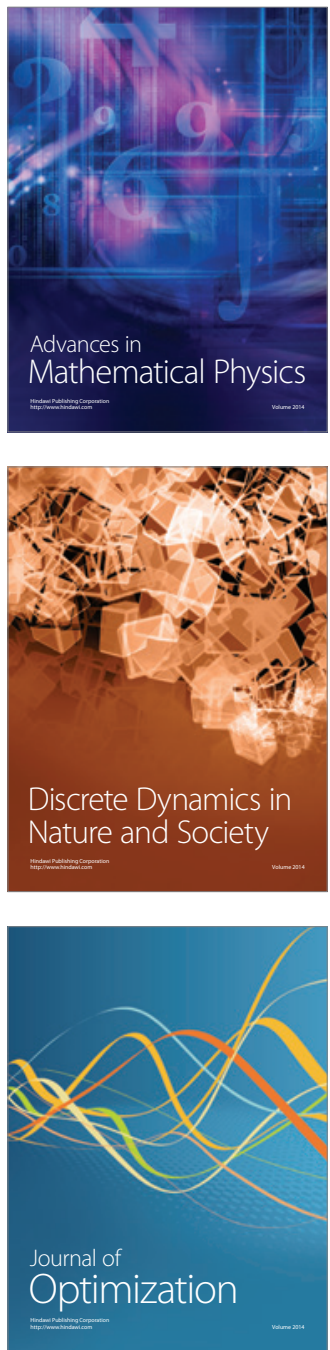